\title{
Partial Cloaking of a Gold Particle by a Single Molecule
}

\author{
Johannes Zirkelbach $\odot,{ }^{1}$ Benjamin Gmeiner $\odot,{ }^{1}$ Jan Renger $\odot,{ }^{1}$ Pierre Türschmann $\odot,{ }^{1}$ Tobias Utikal, ${ }^{1}$ \\ Stephan Götzinger, ${ }^{2,1,3}$ and Vahid Sandoghdar ${ }^{1,2, *}$ \\ ${ }^{1}$ Max Planck Institute for the Science of Light, Erlangen D-91058, Germany \\ ${ }^{2}$ Department of Physics, Friedrich-Alexander University Erlangen-Nürnberg, Erlangen D-91058, Germany \\ ${ }^{3}$ Graduate School in Advanced Optical Technologies (SAOT), Friedrich-Alexander University Erlangen-Nürnberg, \\ Erlangen D-91052, Germany
}

(Received 7 April 2020; accepted 31 July 2020; published 3 September 2020)

\begin{abstract}
Extinction of light by material particles stems from losses incurred by absorption or scattering. The extinction cross section is usually treated as an additive quantity, leading to the exponential laws that govern the macroscopic attenuation of light. In this Letter, we demonstrate that the extinction cross section of a large gold nanoparticle can be substantially reduced-i.e., the particle becomes more transparent—if a single molecule is placed in its near field. This partial cloaking effect results from a coherent plasmonic interaction between the molecule and the nanoparticle, whereby each of them acts as a nanoantenna to modify the radiative properties of the other.
\end{abstract}

DOI: 10.1103/PhysRevLett.125.103603

Macroscopic objects cast a shadow in a beam of light, and the shadow becomes darker if the medium is made optically thicker. This scenario also persists in the nanoscopic domain when the object is smaller than the wavelength of light. For instance, a gold nanoparticle (GNP) of diameter less than $100 \mathrm{~nm}$ can extinguish more than half the power of a laser beam if placed in its focus [1,2]. According to the Beer-Lambert law, this shadow becomes exponentially darker as more particles are added [3]. However, it turns out that one can make a GNP transparent to light by adding a single atom [4-6]. The underlying mechanism of this intriguing phenomenon is the interference between the fields scattered by two near-field coupled oscillators [6-9]. Here, it is helpful to recall that the cross section of a two-level atom with transition at wavelength $\lambda$ can be as large as $\sigma_{0}=3 \lambda^{2} / 2 \pi[10]$, which can be comparable to the extinction cross section and the physical size of a nanoparticle [3]. In other words, although both an atom and a GNP can individually extinguish a laser beam, their composite entity becomes transparent due to a coherent interference effect [6].

Over the past fifteen years, plasmonic platforms have been employed in a wide range of studies [11,12]. One of the main thrusts of these works has been in the incoherent enhancement of excitation or fluorescence rates, where a

Published by the American Physical Society under the terms of the Creative Commons Attribution 4.0 International license. Further distribution of this work must maintain attribution to the author(s) and the published article's title, journal citation, and DOI. Open access publication funded by the Max Planck Society. metallic nanostructure acts as an optical antenna for ameliorating the efficiency of interaction between the emitter and propagating photonic modes [12]. Coupling of plasmonic nanoparticles to emitters has also been theoretically proposed for enhancing light absorption or transmission [4-6,13,14]. Furthermore, there have been the first reports of coherent plasmonic interactions, reaching the strong coupling regime of cavity quantum electrodynamics (CQED) [15-20]. The degree of coherence achieved in these experiments, however, has been very limited because they were performed at room temperature. Recent impressive experiments at cryogenic temperatures have not reached the intrinsic degree of coherence given by the radiative lifetime of the excited state either [21].

Several factors make a laboratory demonstration of plasmonic interactions with long coherence times challenging. First, an emitter and a GNP would have to be placed at separations much smaller than a wavelength. Second, the orientation of the emitter dipole moment has to suit the geometrical features of the nanostructure. Despite decades of nanotechnology experience, these challenges are still not easy to tackle. Third, $\sigma_{0}$ is lowered and the homogeneous spectra are broadened by about five orders of magnitude for solid-state emitters at room temperature. In this Letter, we use dye molecules at a temperature of $T=1.5 \mathrm{~K}$ to essentially reach their natural linewidth limit [22]. We discuss how we overcome various experimental difficulties to achieve a successful realization of emitterinduced transparency of a GNP, analogous to its CQED equivalent using cavities [23,24], and validate our measurements using a theoretical model.

Figure 1(a) illustrates the schematics of the core of our experimental arrangement, where a laser beam is tightly 
(a)

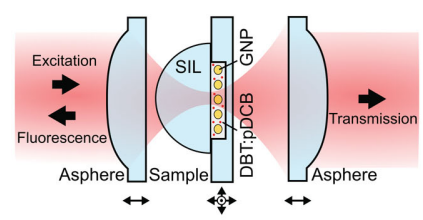

(b)

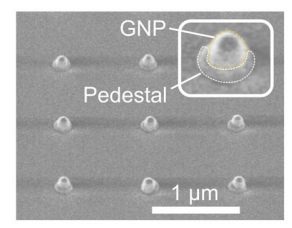

(c)

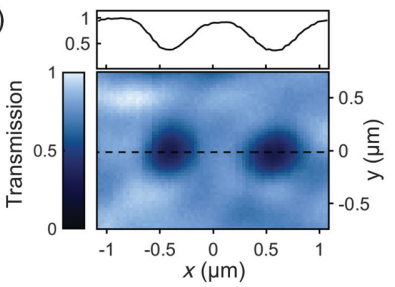

(d)
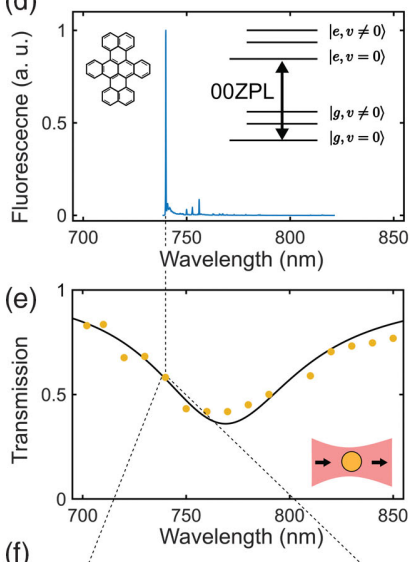

(f)

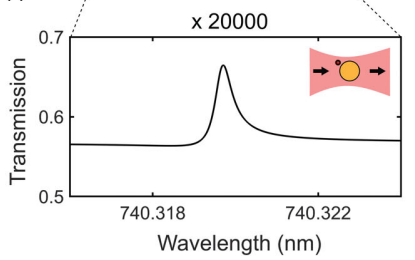

FIG. 1. (a) Schematics of the experimental arrangement. The sample consists of DBT molecules in a pDCB crystal surrounding an array of gold nanoparticles prepared in a nanochannel (see text for details). SIL: solid-immersion lens, GNP: gold nanoparticle. Arrows at the bottom show the translational degrees of freedom. (b) Scanning electron microscope image of the GNP array. Inset: enlargement of a single GNP. (c) Optical transmission image of two GNPs at $\lambda=740 \mathrm{~nm}$. Upper panel shows a normalized cross section along the dashed line. (d) Fluorescence spectrum of DBT recorded upon excitation via transition from $|g, v=0\rangle$ to $|e, v \neq 0\rangle$. The strong emission line at $\lambda=740.3 \mathrm{~nm}$ represents the 00ZPL. Inset: molecular structure and Jablonski diagram of DBT. (e) Plasmon resonance of a GNP measured in transmission (dots) fitted by a Lorentzian profile (black curve). (f) Predicted reduction of the GNP extinction at the resonance of a single molecule that is coupled to it in the near field.

focused onto a sample carrying GNPs and dibenzoterrylene (DBT) molecules. These are placed inside a channel of width $250 \mu \mathrm{m}$ and depth $245 \mathrm{~nm}$ fabricated in a glass chip and covered by a $\mathrm{ZrO}_{2}$ solid-immersion lens (SIL) with a diameter of $3 \mathrm{~mm}$. Figure 1(b) shows an electron microscope image of an array of GNPs with a diameter of about $130 \mathrm{~nm}$, a height of about $100 \mathrm{~nm}$, and a spacing of $1 \mu \mathrm{m}$. The GNPs are fabricated using electron beam lithography on evaporated gold films, followed by etching and subsequent annealing, whereby the etch process is controlled to create glass pedestals of height $35 \mathrm{~nm}$ under the GNPs [see Fig. 1(b) and the Supplemental Material [25] ]. A thin organic crystal of para-dichlorobenzene (pDCB) lightly doped with DBT molecules is prepared to surround the GNP array by first introducing it into the channel in a molten state and next letting it solidify [35] (see also the Supplemental Material [25]). A $5 \mathrm{~nm}$ layer of $\mathrm{Al}_{2} \mathrm{O}_{3}$ is coated on the nanostructures by atomic layer deposition to provide a minimum separation between the DBT molecules

and the gold to avoid strong quenching. The sample is then placed in a cryostat and cooled to $T=1.5 \mathrm{~K}$.

A beam from a Ti:sapphire laser is coupled to the sample via an aspheric lens and the SIL, reaching a focus spot with a full width at half-maximum (FWHM) of $270 \mathrm{~nm}$ assessed by mapping the fluorescence of a single molecule. A second aspheric lens is used to recollimate the laser beam in transmission. Figure 1(c) displays a transmission image recorded by scanning the focus of the incident laser beam across two GNPs. A cut through the image (see upper panel) reveals an extinction dip of about $50 \%$ from each GNP. In Fig. 1(e), we plot the extinction plasmon spectrum of a single GNP (see the Supplemental Material [25]).

The inset in Fig. 1(d) displays the structure of DBT and its Jablonski diagram. DBT is a member of the polycyclic aromatic hydrocarbon family and possess a strong zerophonon line (00ZPL) between $|g, v=0\rangle$ (ground electronic and vibrational state) and $|e, v=0\rangle$ (ground vibrational and electronic excited state) when placed in an appropriate crystal. Figure 1(d) presents the emission spectrum of a single DBT molecule upon excitation to a $|e, v \neq 0\rangle$ state, followed by a fast nonradiative decay to $|e, v=0\rangle$ with a radiative lifetime of a few nanoseconds. The spectrum shows that a large fraction of the decay from $|e, v=0\rangle$ takes place via the 00ZPL, leading to a branching ratio of about $44 \%$ [36].

In our sample, DBT molecules are stochastically distributed in the pDCB matrix, but we can identify and interrogate each molecule individually with very high spatial and spectral resolution. Here, we first scan the wavelength of the narrow-band laser across the inhomogeneous band of DBT:pDCB around $\lambda=740 \mathrm{~nm}[35,36]$. The exquisitely narrow 00ZPL resonances $(20-40 \mathrm{MHz})$ associated with the molecules do not overlap so that each can be selectively addressed by tuning the laser frequency. The spectral selection of a single molecule also allows us to image it and, thus, determine the center of its point-spread function beyond the diffraction limit.

Our goal in this Letter is to show that a single molecule can counteract the extinction effect of a single GNP. Our strategy is first, to locate a DBT molecule close to a GNP and examine its near-field coupling via incoherent fluorescence measurements. We then explore the coherent effect of the composite system of a molecule and a GNP. As depicted in Fig. 1(f), we expect the resonant transmission signal to experience a substantial increase.

To identify molecules that are located in the near field of a GNP, we first centered the focus of the laser beam on the GNP and scanned the laser frequency. Figure 2(a) presents an example of the ZPLs obtained at this position by recording the red-shifted fluorescence as the laser frequency was scanned through the inhomogeneous band of about $1 \mathrm{THz}$. The differences in the observed signal stem mostly from variations in the positions of the molecules within the laser intensity profile. The inset in Fig. 2(a) 

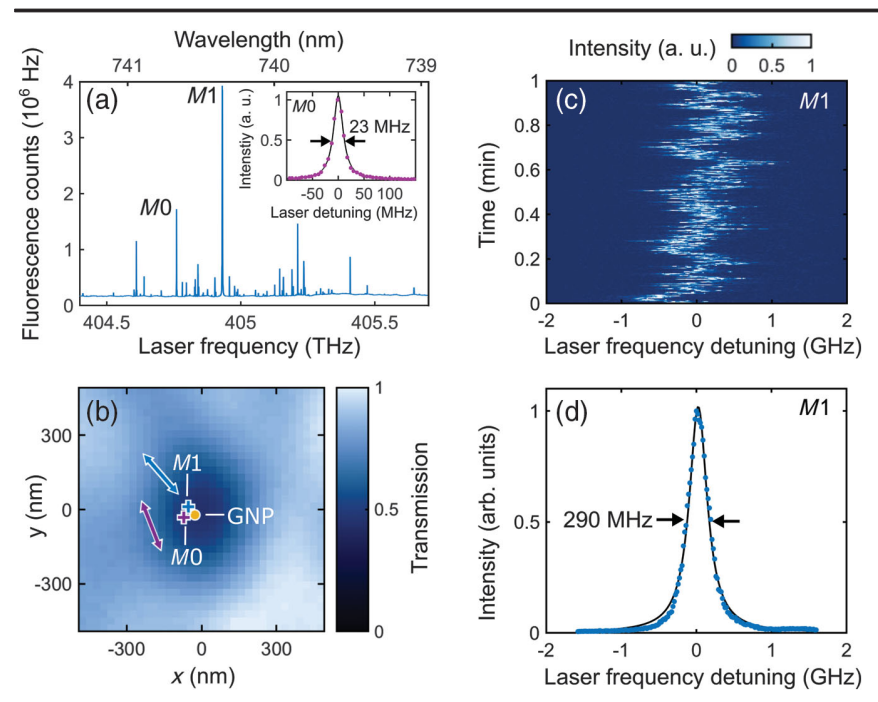

FIG. 2. (a) Fluorescence of DBT molecules located within a focal spot of one GNP as a function of the excitation laser frequency. The applied laser power of $79 \mathrm{nW}$ corresponds to an excitation intensity above saturation. Inset: an enlargement of the spectrum of $M 0$ recorded at low excitation power. (b) An extinction image of a single GNP recorded in transmission overlaid with the locations of the GNP obtained from its fluorescence image (yellow), $M 0$ (magenta), and $M 1$ (blue). The arrows depict the in-plane orientations of the molecular dipole moments. (c) 240 spectra recorded at $20 \mathrm{GHz} / \mathrm{s}$ over one minute well below saturation (excitation power $0.04 \mathrm{nW}$ ). (d) Sum of the aligned spectra shown in (c) (symbols) and a Lorentzian fit (solid curve).

displays an enlargement of one of the strongest ZPL resonances (marked as $\mathrm{M} 0$ ) with a linewidth of $23 \mathrm{MHz}$ measured at low excitation power. This spectrum is consistent with those reported in bulk crystals [36] and represents a typical spectral response of the molecules that are not coupled to GNPs in our current sample.

We determined the lateral positions of the molecules associated with the different resonances in Fig. 2(a) at a precision of about $10 \mathrm{~nm}$ by fitting Gaussian functions to their point-spread functions [37]. In addition, we applied this method to the fluorescence image of the GNP [38,39]. The yellow circle and the magenta cross in Fig. 2(b) mark the lateral positions of the GNP and $M 0$, respectively, but it should be borne in mind that the results can contain systematic errors due to the redirection of the molecular emission by the GNP [40-42].

Molecules that experience a significant degree of plasmonic enhancement are expected to display shorter fluorescence lifetimes, broader 00ZPLs and higher emission rates upon saturation. The channel depth of $245 \mathrm{~nm}$ limits the axial distance variations and greatly simplifies the search for such GNP-coupled molecules. In what follows, we investigate one coupled molecule, which we label $M 1$, in great detail. The blue cross in Fig. 2(b) marks the apparent center of $M 1$ overlaid on the extinction image of the GNP. We determined the orientation of the
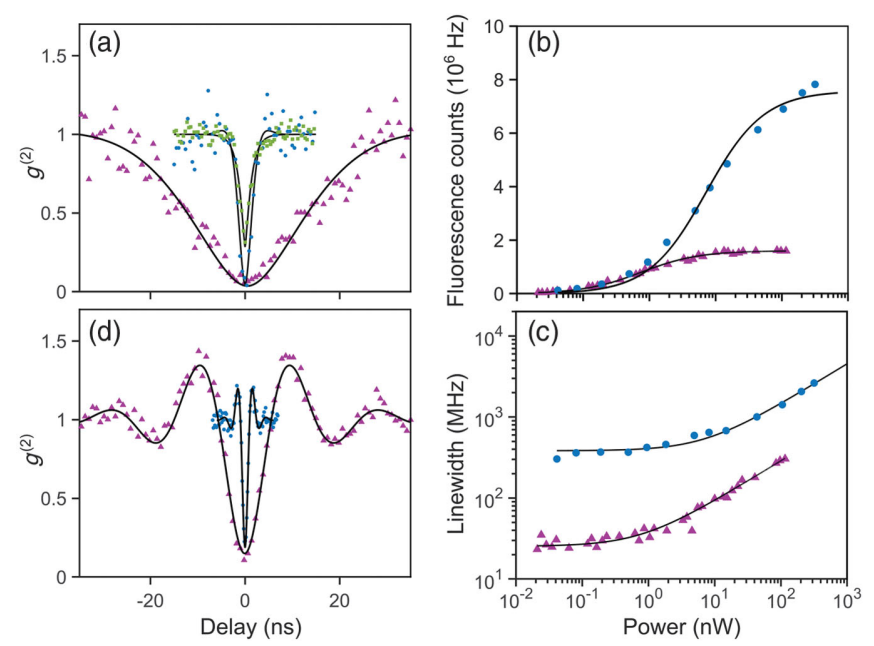

FIG. 3. (a) Second-order autocorrelation function, $g^{(2)}(\tau)$ for $M 0$ excited via 00ZPL (magenta), $M 1$ excited via 00ZPL (blue), and $M 1$ excited via a higher vibrational level $|e, v \neq 0\rangle$ (green). (b) Fluorescence signal versus excitation power for $M 0$ (magenta) and $M 1$ (blue) under 00ZPL excitation. (c) 00ZPL linewidth (FWHM) versus excitation power extracted from the spectra used in (b). (d) Same as in (a) but excited above saturation. Solid black lines are fits to the data (see the Supplemental Material [25]).

molecular transition dipole moment by adjusting the polarization of the excitation light to maximize its fluorescence signal. We found the in-plane dipole moment of $M 1$ to point nearly radially towards the GNP (considered to be spherical) whereas the dipole moment of $M 0$ was tangential to the GNP [see Fig. 2(b) and the Supplemental Material [25] ].

Repeated fluorescence excitation spectra in Fig. 2(c) reveal that $M 1$ experienced spectral instabilities over a time scale of seconds. Indeed, spectral diffusion poses one of the great challenges in performing high-resolution coherent studies in the near field of plasmonic structures because the crystallinity of solid-state matrices, be it semiconductor, inorganic or organic is compromised at an interface between different materials and geometries. To get around the spectral jitter, we scanned the laser frequency at a fast rate of $20 \mathrm{GHz} / \mathrm{s}$. The blue symbols in Fig. 2(d) show the average of 240 such scans obtained after aligning their midpoints determined as the center of the measured FWHMs in each scan. A good Lorentzian fit (black curve) indicates that our procedure successfully accounts for the slow spectral diffusion. We also applied a similar analysis to account for the residual spectral diffusion that occurred in some measurements on $M 0$.

The extracted FWHM of $290 \mathrm{MHz}$ is much broader than that of $M 0$ and provides a first indication for plasmonic coupling. To examine this further, we measured fluorescence intensity correlations in a Hanbury-Brown and Twiss arrangement. The magenta triangles in Fig. 3(a) display $g^{(2)}(\tau)$ for $M 0$ excited via its 00ZPL, while the blue and green dots show the outcome for $M 1$ excited via its 00ZPL 
and a higher vibrational level of the electronic excited state $|e, v \neq 0\rangle$, respectively. Pronounced antibunching effects at zero delay time assure us that the signals stem from single molecules. A fit to the measured data lets us extract an excited state lifetime of $T_{1}=8.1 \pm 0.4$ ns for $M 0$ and $T_{1}=$ $1.4 \pm 0.1 \mathrm{~ns}$ for $M 1$ (see the Supplemental Material [25]).

The resulting sixfold lifetime shortening could be caused by an increase in the nonradiative rate $\left(\Gamma_{\mathrm{nr}}\right)$ or the enhancement of the radiative rate $\left(\Gamma_{\mathrm{r}}\right)$, i.e., a plasmonic Purcell effect $[12,43]$. To inquire about the relative weights of these effects, we excited $M 0$ and $M 1$ via their 00ZPLs at different incident powers. The fluorescence signals presented in Fig. 3(b) show that at saturation, the power radiated by $M 1$ is about five times larger than that of $M 0$ if we assume similar collection efficiencies [44]. This confirms a substantial Purcell enhancement of the radiative decay. Figure 3(c) also presents the evolution of the molecular linewidths as a function of the laser power.

Our findings verify that a GNP acts as a plasmonic nanoantenna to enhance the radiative properties of $M 1$. However, as in the case of the great majority of previous reports on plasmonic antennas $[11,12,43,45]$, our abovementioned studies were solely based on the behavior of the excited-state population observed via the red-shifted fluorescence. Resonant scattering, however, depends sensitively on the degree of coherence in the molecular dipole moment. Indeed, the measured fluorescence lifetime of 1.4 ns lets us deduce a homogeneous linewidth $\left(\Gamma_{1} / 2 \pi\right)$ of $114 \pm 8 \mathrm{MHz}$ for the 00ZPL of $M 1$, which is notably less than the directly measured FWHM of $290 \mathrm{MHz}$. Thus, we expect a contribution from pure dephasing.

To extract additional information about the fast dynamics that might contribute to dephasing, we analyzed $g^{(2)}(\tau)$ as a function of excitation power. Figure 3(d) plots two examples of such measurements for $M 0$ (magenta) and $M 1$ (blue) under strong excitation. A simultaneous fit of the data let us extract a pure dephasing rate of $\Gamma^{\star} / 2 \pi=87 \pm 35 \mathrm{MHz}$ for $M 1$, corresponding to $T_{2}^{\star} \sim 1.8 \mathrm{~ns}$ (see the Supplemental Material [25] for a detailed discussion). This implies $\mathrm{FWHM}=$ $\left(\Gamma_{1}+2 \Gamma_{2}^{\star}\right) / 2 \pi=(114+2 \times 87) \mathrm{MHz}=288 \mathrm{MHz}$, which is in good agreement with the directly measured value of $290 \mathrm{MHz}$.

Having established a good understanding of the degree of coherence in our molecular system, we now present our results on resonant extinction spectra. The magenta data points in Fig. 4(a) display the transmitted power of the incident laser beam as its frequency was scanned through the 00ZPL of $M 0$, whereby the data are normalized to the GNP extinction signal [see Figs. 1(c), 1(e), and 1(f) and the Supplemental Material [25] ]. The observed extinction dip of $4 \%$ is in the range of previous measurements on single polycyclic aromatic hydrocarbon molecules [46,47]. However, the blue symbols in Fig. 4(a) show that in the case of $M 1$, in addition to a larger linewidth, the transmitted
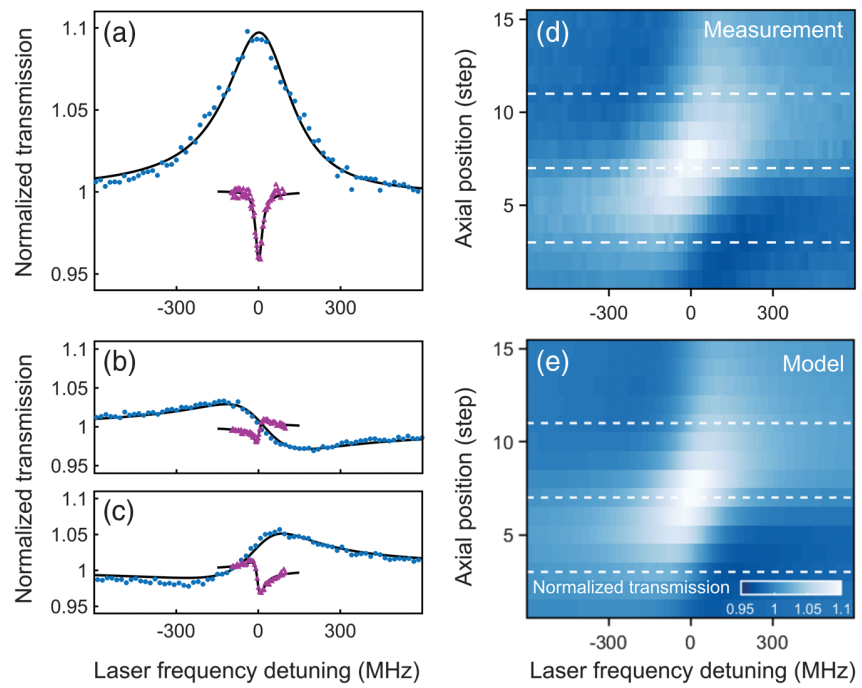

FIG. 4. (a) Transmission spectra recorded about the 00ZPL resonances of $M 1$ (blue) and $M 0$ (magenta) placed in the focus of the excitation beam, corresponding to the middle dashed cut in (d). (b),(c) Same as in (a) but for molecules placed on two opposite sides of the focus, as indicated by the lower and upper dashed cuts in (d). (d) Series of transmission scans through the 00ZPL resonance of $M 1$ at 15 different axial positions of the sample over an estimated total distance of $3.5 \mu \mathrm{m}$ (see the Supplemental Material [25]). All data in this figure were recorded in the weak excitation regime [0.04 nW, see Figs. 3(b) and 3(c)]. (e) Calculated transmission spectra to fit the data in (d). See the Supplemental Material [25] for details.

power is increased by $10 \%$. In other words, by adding a single molecule, we have indeed turned a gold nanoparticle more transparent.

Because extinction is intrinsically an interferometric phenomenon [10], the laser intensity observed in the far field depends on the relative phase and amplitude of the excitation and scattered fields at the detector. Thus, considering the characteristic dependence of the Gouy phase around the focal plane, one expects a clear change in the resonance profile upon axial scan of the sample [48]. The Fano-type spectra in Figs. 4(b) and 4(c) show this effect for both $M 0$ (magenta) and $M 1$ (blue) at two positions of the sample-SIL assembly across the focal plane of the aspherical lens [see Fig. 1(a)]. In Fig. 4(d) we plot the extinction spectra of $M 1$ for an extended axial sweep. A more detailed discussion and the equivalent data for $M 0$ can be found in the Supplemental Material [25].

The quantitative details of the plasmonic coupling of $M 1$ crucially depend on the geometrical features of the GNP as well as the exact position and orientation of the molecule. Considering that we do not have access to these parameters, we cannot use rigorous numerical simulations to fit our experimental data. However, the underlying physics can be captured by employing a model based on driven coupled oscillators [8,9,49] (see the Supplemental Material [25] for details). Figure 4(e) presents the outcome of such 
calculations fitted to the corresponding measurements shown in Fig. 4(d). The solid curves in Figs. 4(a)-4(c) through the measured data of $M 1$ represent the cuts marked by the dashed lines in Fig. 4(e). The consistent agreement between the experimental data and the theoretical model provides assurance that the resonance profiles follow the phase behavior expected from the coherent interaction between the laser beam, GNP and $M 1$. This analysis lets us deduce a coupling constant of $2 g / 2 \pi=53 \mathrm{GHz}$ and a cooperativity of $C=\left(4 g^{2} / \kappa \gamma\right)=0.23$, as commonly stated in the CQED formalism. It is worth emphasizing that the reason for $C$ reaching the order of unity in our arrangement is the high degree of emitter coherence (i.e., small $\gamma$ ), but we remain deep in the weak coupling regime since $4 g \ll$ $(\kappa+\gamma)[20]$ (see the Supplemental Material [25]). We also note that our model also predicts a "Lamb shift" of $12 \mathrm{MHz}$ in the resonance of $M 1$ induced by coupling to the GNP. While this small frequency shift is accessible to our high-resolution spectroscopic studies, we did not verify it because we could not examine $M 1$ in the absence of GNP as is done in scanning-probe arrangements $[15,16,18,43,45]$.

Two main advantages of plasmonic antennas over conventional microresonators regard their nanoscopic compactness and broad bandwidth. These features provide important opportunities for the realization of subwavelength building blocks of quantum photonic circuits for operations such as switching and phase modulation. In future, stronger nearfield couplings through more advanced antenna designs $[12,19,45]$ will enhance the system performance well beyond the first demonstration presented in this Letter.

We thank Jaesuk Hwang and Andreas Maser for their very early experimental efforts on the project. This work was supported by an Alexander von Humboldt professorship and the Max Planck Society.

*Corresponding author vahid.sandoghdar@mpl.mpg.de

[1] N. Mojarad, G. Zumofen, V. Sandoghdar, and M. Agio, J. Eur. Opt. Soc. 4, 09014 (2009).

[2] M. Celebrano, R. Lettow, P. Kukura, M. Agio, A. Renn, S. Götzinger, and V. Sandoghdar, Opt. Express 18, 13829 (2010).

[3] C. F. Bohren and D. R. Huffman, Absorption and Scattering of Light by Small Particles (Wiley, New York, 1983).

[4] X. Wu, S. K. Gray, and M. Pelton, Opt. Express 18, 23633 (2010).

[5] A. Ridolfo, O. Di Stefano, N. Fina, R. Saija, and S. Savasta, Phys. Rev. Lett. 105, 263601 (2010).

[6] X.-W. Chen, V. Sandoghdar, and M. Agio, Phys. Rev. Lett. 110, 153605 (2013).

[7] B. Luk'yanchuk, N. I. Zheludev, S. A. Maier, N. J. Halas, P. Nordlander, H. Giessen, and C. T. Chong, Nat. Mater. 9, 707 (2010).
[8] B. Gallinet, in Fano Resonances in Optics and Microwaves, edited by E. Kamenetskii, A. Miroshnichenko, and A. Sadreev (Springer, New York, 2018), pp. 109-136.

[9] F. Ruesink, H. M. Doeleman, E. Verhagen, and A. F. Koenderink, Phys. Rev. Lett. 120, 206101 (2018).

[10] G. Zumofen, N. M. Mojarad, V. Sandoghdar, and M. Agio, Phys. Rev. Lett. 101, 180404 (2008).

[11] A. F. Koenderink, ACS Photonics 4, 710 (2017).

[12] V. Sandoghdar, M. Agio, X.-W. Chen, S. Götzinger, and K.-G. Lee, in Optical Antennas, edited by A. Alùand M. Agio (Cambridge University Press, Cambridge, 2013), pp. 100-121.

[13] W. Zhang, A. O. Govorov, and G. W. Bryant, Phys. Rev. Lett. 97, 146804 (2006).

[14] R. D. Artuso and G. W. Bryant, Nano Lett. 8, 2106 (2008).

[15] T. Hartsfield, W.-S. Chang, S.-C. Yang, T. Ma, J. Shi, L. Sun, G. Shvets, S. Link, and X. Li, Proc. Natl. Acad. Sci. U.S.A. 112, 12288 (2015).

[16] H. Groß, J. M. Hamm, T. Tufarelli, O. Hess, and B. Hecht, Sci. Adv. 4, eaar4906 (2018).

[17] H. Leng, B. Szychowski, M.-C. Daniel, and M. Pelton, Nat. Commun. 9, 4012 (2018).

[18] K.-D. Park, M. A. May, H. Leng, J. Wang, J. A. Kropp, T. Gougousi, M. Pelton, and M. B. Raschke, Sci. Adv. 5, eaav5931 (2019).

[19] R. Chikkaraddy, B. de Nijs, F. Benz, S. J. Barrow, O. A. Scherman, E. Rosta, A. Demetriadou, P. Fox, O. Hess, and J. J. Baumberg, Nature (London) 535, 127 (2016).

[20] M. Pelton, S. D. Storm, and H. Leng, Nanoscale 11, 14540 (2019).

[21] Y. Zhang, Q.-S. Meng, L. Zhang, Y. Luo, Y.-L. Yu, B. Yang, Y. Zhang, R. Esteban, J. Aizpurua, Y. Luo, J.-L. Yang, Z.-C. Dong, and J. G. Hou, Nat. Commun. 8, 15225 (2017).

[22] Decisive advantages of dye molecules in organic matrices are as follows: small size of about one nanometer, availability at many different wavelengths, and strong Fourierlimited zero-phonon lines in the order of 10-50 MHz. This is to compare with the typical room-temperature linewidth of a solid-state emitter at our wavelength, which is about $10 \mathrm{THz}$.

[23] E. Waks and J. Vuckovic, Phys. Rev. Lett. 96, 153601 (2006).

[24] D. Wang, H. Kelkar, D. Martin-Cano, T. Utikal, S. Götzinger, and V. Sandoghdar, Phys. Rev. X 7, 021014 (2017).

[25] See the Supplemental Material at http://link.aps.org/ supplemental/10.1103/PhysRevLett.125.103603 for more details about the sample, the experimental setup, the models used to fit the autocorrelation measurements, the coupled oscillator model, and the axial scan of the sample, which includes Refs. [26-34].

[26] J.-B. Trebbia, H. Ruf, P. Tamarat, and B. Lounis, Opt. Express 17, 23986 (2009).

[27] R. Loudon, The Quantum Theory of Light, 3rd ed. (Oxford University Press, Oxford, 2000).

[28] A. Muller, E. B. Flagg, P. Bianucci, X. Y. Wang, D. G. Deppe, W. Ma, J. Zhang, G. J. Salamo, M. Xiao, and C. K. Shih, Phys. Rev. Lett. 99, 187402 (2007).

[29] S. Grandi, K. D. Major, C. Polisseni, S. Boissier, A. S. Clark, and E. A. Hinds, Phys. Rev. A 94, 063839 (2016). 
[30] F. Stete, W. Koopman, and M. Bargheer, ACS Photonics 4, 1669 (2017).

[31] M.-S. Kim, A. Assafrao, T. Scharf, C. Rockstuhl, S. F. Pereira, H. P. Urbach, and H. P. Herzig, J. Mod. Opt. 60, 197 (2013).

[32] J. T. Hugall, A. Singh, and N. F. van Hulst, ACS Photonics 5, 43 (2018).

[33] B. C. Buchler, T. Kalkbrenner, C. Hettich, and V. Sandoghdar, Phys. Rev. Lett. 95, 063003 (2005).

[34] P. Stoller, V. Jacobsen, and V. Sandoghdar, Opt. Lett. 31, 2474 (2006).

[35] B. Gmeiner, A. Maser, T. Utikal, S. Götzinger, and V. Sandoghdar, Phys. Chem. Chem. Phys. 18, 19588 (2016).

[36] N. R. Verhart, M. Müller, and M. Orrit, ChemPhysChem 17, 1524 (2016).

[37] S. Weisenburger and V. Sandoghdar, Contemp. Phys. 56, 123 (2015).

[38] Y. Fang, W.-S. Chang, B. Willingham, P. Swanglap, S. Dominguez-Medina, and S. Link, ACS Nano 6, 7177 (2012).

[39] Y. Cheng, G. Lu, Y. He, H. Shen, J. Zhao, K. Xia, and Q. Gong, Nanoscale 8, 2188 (2016).

[40] L. Su, H. Yuan, G. Lu, S. Rocha, M. Orrit, J. Hofkens, and H. Uji-i, ACS Nano 10, 2455 (2016).
[41] B. Fu, B. P. Isaacoff, and J. S. Biteen, ACS Nano 11, 8978 (2017).

[42] G. Blanquer, B. van Dam, A. Gulinatti, G. Acconcia, Y. De Wilde, I. Izeddin, and V. Krachmalnicoff, ACS Photonics 7, 393 (2020).

[43] S. Kühn, U. Håkanson, L. Rogobete, and V. Sandoghdar, Phys. Rev. Lett. 97, 017402 (2006).

[44] This is a reasonable assumption because the two molecules are very close to each other and the numerical aperture of our collection optics is very high.

[45] K. Matsuzaki, S. Vassant, H.-W. Liu, A. Dutschke, B. Hoffmann, X. Chen, S. Christiansen, M. R. Buck, J. A. Hollingsworth, S. Götzinger, and V. Sandoghdar, Sci. Rep. 7, 42307 (2017).

[46] G. Wrigge, I. Gerhardt, J. Hwang, G. Zumofen, and V. Sandoghdar, Nat. Phys. 4, 60 (2008).

[47] We observed extinction dips as large as $10 \%$ for other molecules in our sample. The lower extinction effect is because the GNP in this study did not lie on the SIL axis.

[48] J. Hwang and W. Moerner, Opt. Commun. 280, 487 (2007).

[49] C. Garrido Alzar, M. Martinez, and P. Nussenzveig, Am. J. Phys. 70, 37 (2002). 